\title{
RQA correlations on real business cycles time series
}

\author{
GIUSEPPE ORLANDO ${ }^{1,2, *}$ and GIOVANNA ZIMATORE ${ }^{3}$ \\ ${ }^{1}$ Università degli Studi di Bari "Aldo Moro" - Department of Economics and Finance, Via C. Rosalba 53, Bari, \\ I-70124 Italy \\ ${ }^{2}$ Università degli Studi di Camerino - School of Science and Technologies, Via M. delle Carceri 9, Camerino, \\ I-62032 Italy \\ ${ }^{3}$ CNR-IDASC, Institute of Acoustics and Sensors "O. M. Corbino", Via del Fosso del Cavaliere 100, Roma, \\ I-00133 Italy \\ *Corresponding author. E-mail: giuseppe.orlando@uniba.it and giuseppe.orlando@unicam.it
}

\begin{abstract}
Trade cycles are complex phenomena which oscillate because of economic downturns and expansions. Recurrence quantification analysis (RQA) detects state changes without necessitating any a priori mathematical assumption and highlights hidden features of the dynamics both at equilibrium and near transition phases. This paper aims to understand some potential application of recurrence quantification analysis in detecting recessions.
\end{abstract}

Keywords. Determinism; numerical chaos; applications to economics; economic dynamics.

PACS Nos 05.10.-a; 05.45.Tp; 05.45.-a

\section{Introduction}

This work concerns the application of recurrence plots and of their quantitative description provided by recurrence quantification analysis (RQA) in order to understand subtle but physiologically relevant changes in the dynamic regime of business time series. RQA aims at a direct and quantitative appreciation of the amount of deterministic structure of time series and has been shown to be an efficient and relatively simple tool in nonlinear analysis of a wide class of signals. The technique allows for the identification of sudden phase changes possibly pointing to mechanistically relevant phenomena. Therefore, RQA may be suitable to study business cycles and could potentially be used for early detection of recessions.

The paper is organized as follows. Section 2 contains the definition of business cycle and summarizes the literature on recurrence quantification analysis and its applications to economics and finance. Section 3 shows the analysis performed and the results obtained. A final section draws some concluding remarks, and suggestions for future research.

\section{Literature review}

According to The National Bureau of Economic Research (NBER) [1], a recession is "a significant decline in economic activity spread across the economy, lasting more than a few months, normally visible in real GDP, real income, employment, industrial production, and wholesale-retail sales. A recession begins just after the economy reaches a peak of activity and ends as the economy reaches its trough. Between trough and peak, the economy is in an expansion. Expansion is the normal state of the economy; most recessions are brief and they have been rare in recent decades" (see figure 1).

The ability of RQA to predict catastrophic changes is in line with the fact that RQA is based upon the change in correlation structure of the observed phenomenon known to precede the actual event in many different systems ranging from physiology [2] and geophysics [3] to economy [4]. Gorban et al. [5] found that even before crisis, correlation increases as does variance (and volatility). In particular their dataset, composed of the thirty largest companies from the UK stock market in the period 2006-2008, supports the hypothesis of increasing correlations during a crisis and, therefore, that correlation (or equivalently determinism) increases when the market goes down (respectively decreases when it recovers).

RQA in economics started relatively recently [6-10]. Fabretti and Ausloos [11] found cases where RQA could detect a warning before a crash. In accordance to that, Addo et al. [12] assert "the usefulness of recurrence plots in identifying, dating and explaining financial 


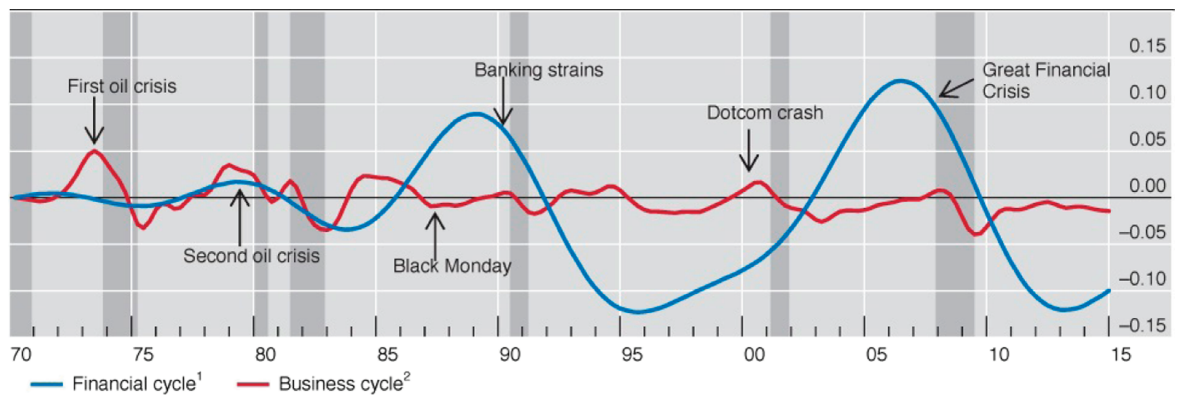

Figure 1. BIS 85th Annual Report 2015.

bubbles and crisis". For Strozzi et al. [13] determinism and laminarity "changes more clearly than standard deviation and then they provide an alternative measure of volatility". Finally, quoting Piskun et al. [14], laminarity (LAM) "is the most suitable measure, sensitive to critical events on markets".

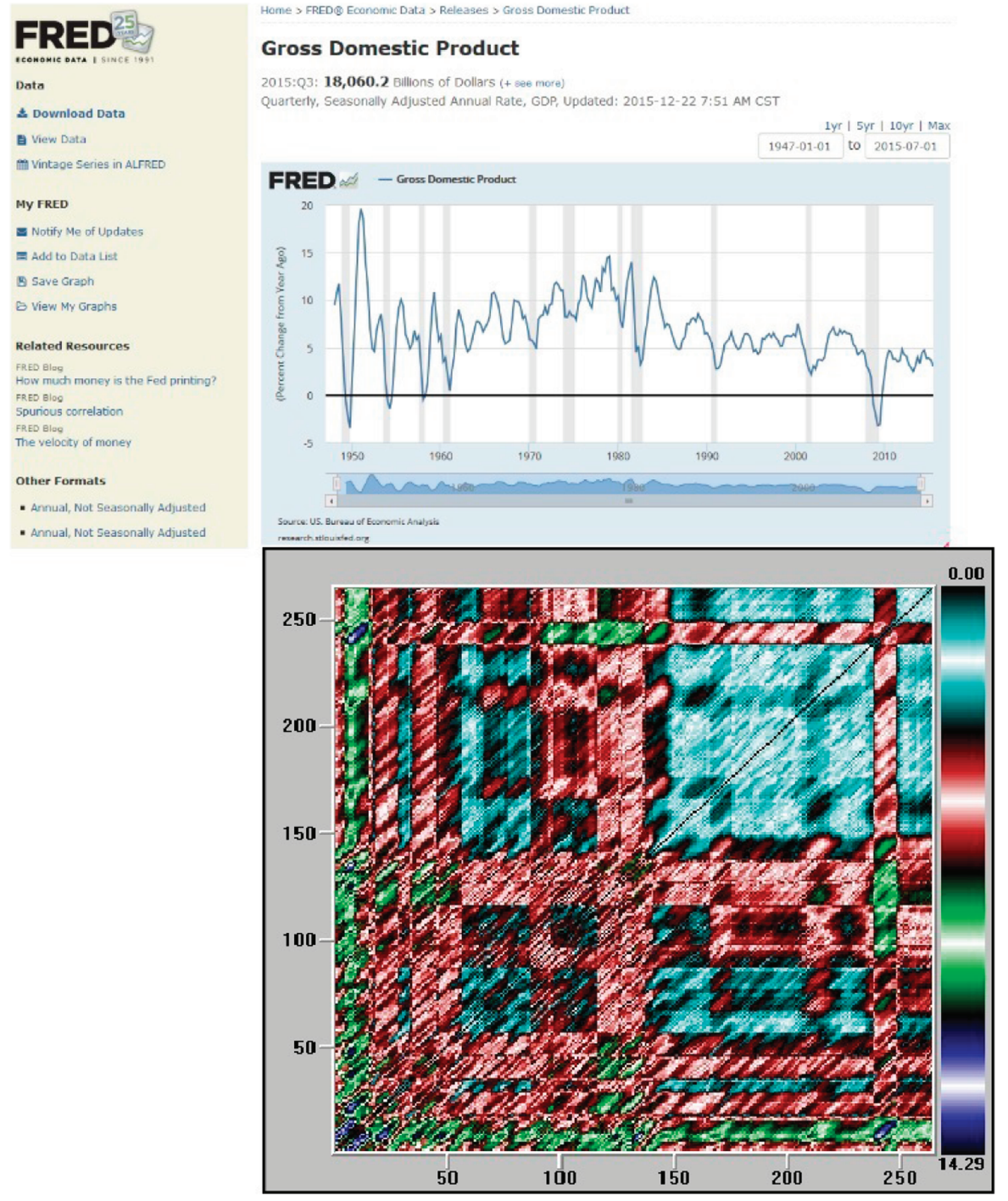

Figure 2. Changes in US GDP (above) and its unthreshold RP or Distance Matrix (DM) (below). Period: 01-01-1947 01-07-2015 (Source: St. Louis Fed, FRED database.) 


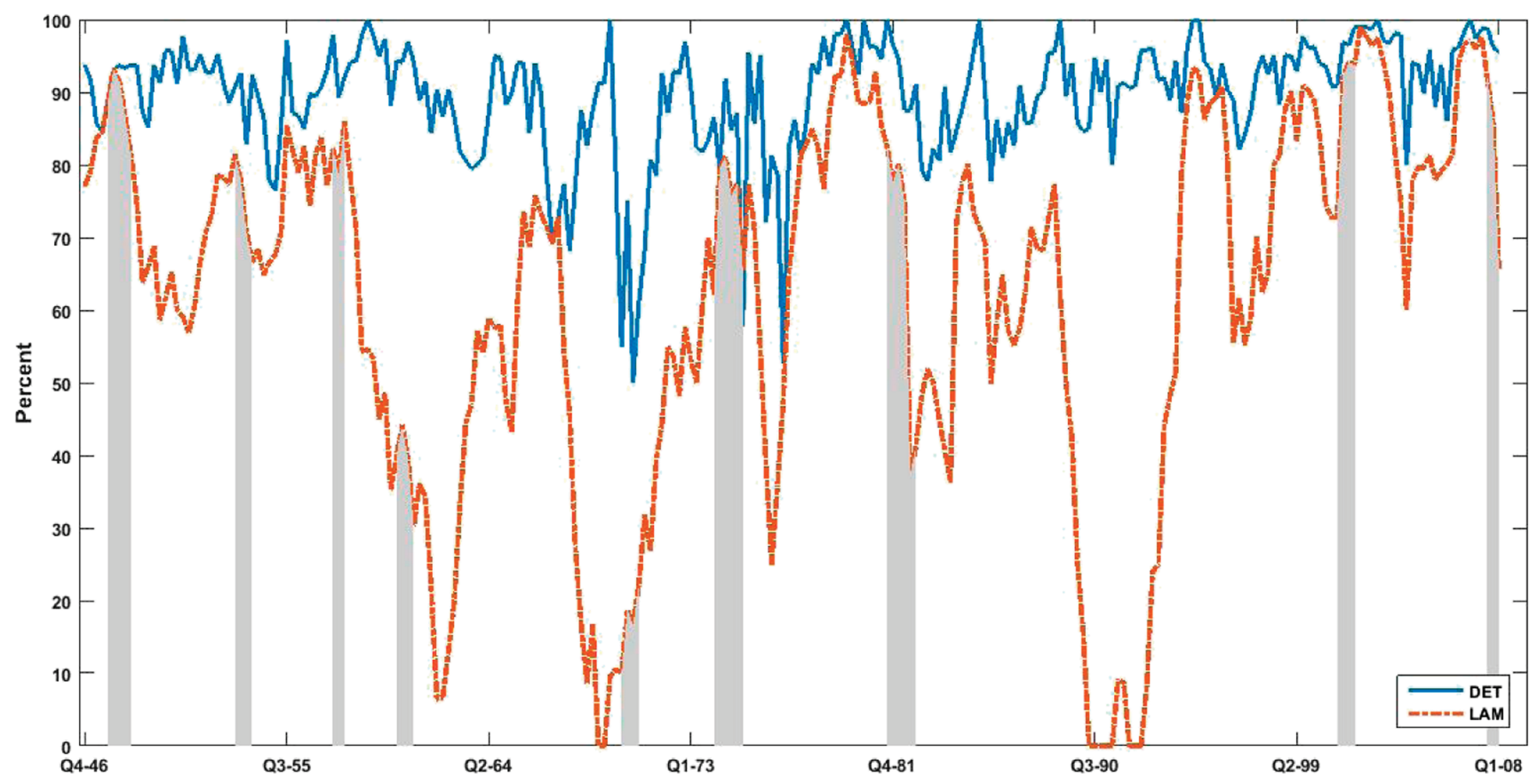

Figure 3. Dynamical analysis in the sliding window mode (RQE) where percent of laminarity (LAM) and percent of determinism (DET) refer to the same time series as in figure 2. Overlapping sliding windows of 50 days length (50 data points) shifted by 1 day (49 data-point overlap) were taken. Variables are plotted in central position in standardized units (su), i.e., after subtracting average value from absolute values and dividing by standard deviation in each window.

\section{Results and analysis}

In order to study the business cycles and recessions from the point of view of a phase transition of nonlinear phenomena we applied RQA on time series extracted from different sources such as Federal Reserve Economic Data (FRED) - St. Louis Fed and OECD.

\subsection{Recurrence plot (RP)}

In figure 2 the recurrence plot of USA GDP\% is shown just below the FRED graph. Greyed areas correspond to periods of economic recession as reckoned by FRED. For the unthreshold RP or Distance Matrix $(\mathrm{DM})^{1}$ it is possible to observe the anticipating transitions to turbulent phases. The noticeable result consists in a correspondence between vertical lines in DM (i.e. chaos to chaos transitions) and grey lines (recession periods).

${ }^{1}$ See for example Garcia-Ochoa et al. (2009) [16]. "There are two different types of RPs: unthresholded recurrence plots (UTRP) and thresholded recurrence plots (TRPs). An unthreshold RP is not binary but its matrix $\mathrm{R}_{i j}^{u}$ is given by the (real valued) distances of the vectors $\vec{x}_{i}$ and $\vec{x}_{j}$. The matrix then is usually represented in a two dimensional coloured plot" or Distance Matrix (DM) it is possible to observe the anticipating transitions to turbulent phases. The noticeable result consists in a correspondence between vertical lines in DM (i.e. chaos to chaos transitions) and grey lines (recession periods).

\subsection{Recurrence quantification epoch ( $R Q E)$}

Bastos and Caiado [15] found a reduction in DET and LAM during the sub-prime mortgage crisis, and Fabretti and Ausloos (2005) [11] and Kousik et al. (2010) [17] reported the highest value of DET and LAM during the bullish period. To investigate along this line we applied RQE analysis on real time series. (Parameters: window size $=50$ points; shift $=1$ point; lag $=1$; embedding $=10$; radius $=80$; line $=5$.) In figure 3 it can be observed that

Table 1. USA recessions.

\begin{tabular}{lccc}
\hline \multicolumn{3}{c}{ Recessions } \\
\hline & From & \multicolumn{3}{c}{ To } \\
\hline Quarter & Year & Quarter & Year \\
Q4 & 1948 & Q4 & 1949 \\
Q3 & 1953 & Q1 & 1954 \\
Q4 & 1957 & Q1 & 1958 \\
Q3 & 1960 & Q1 & 1961 \\
Q1 & 1970 & Q4 & 1970 \\
Q1 & 1974 & Q2 & 1975 \\
Q1 & 1980 & Q2 & 1980 \\
Q3 & 1981 & Q4 & 1982 \\
Q3 & 1990 & Q1 & 1991 \\
Q2 & 2001 & Q4 & 2001 \\
Q1 & 2008 & Q3 & 2009 \\
\hline
\end{tabular}

US. Bureau of Economic Analysis [18]. 
Table 2. Intervals and corresponding values for $\mu$ and $\sigma^{2}$.

\begin{tabular}{rrrrr}
\hline N. & \multicolumn{2}{c}{ Interval } & $\mu$ & $\sigma^{2}$ \\
\hline 1 & 0 & 100 & 0 & 1 \\
2 & 101 & 140 & 1 & 1 \\
3 & 141 & 200 & 1 & 4 \\
4 & 201 & 280 & 4 & 4 \\
5 & 281 & 300 & 4 & 6 \\
6 & 301 & 400 & -5 & 6 \\
7 & 401 & 420 & 2 & 6 \\
8 & 421 & 500 & 2 & 1 \\
8 & 501 & 560 & 0 & 1 \\
9 & 561 & 600 & 0 & 3 \\
10 & 601 & 700 & 1 & 3 \\
\hline
\end{tabular}

the fall of DET corresponds to the grey vertical line indicated by FRED DATA (see table 1).

\subsection{RQE correlation index}

Let us define the set of indices $\mathcal{I}=[1, n] \subseteq \mathbb{N}$, and for each $(k, i) \in \mathbb{N}^{*} \times \mathbb{N}^{*}$ with $k<n$ and $i$ $\leq n-k+1$, we define the discrete times for the rolling window $\mathcal{I}_{k, i}=[i, i+k-1](\subset \mathbb{N})$, where $k$ and $i$ are, respectively, the size and the window's index. It follows that the number of windows is $q=n-k+1$ $(\geq 2)$.

For each $(k, i, l) \in \mathbb{N}^{*} \times \mathbb{N}^{*} \times \mathbb{N}^{*}$, we denote the set of RQE times series as $S=\left(S^{(l)}\right)_{1 \leq l \leq L}$, where $S^{(l)}=\left\{S_{t}^{(l)} \mid 1 \leq t \leq n\right\}$ represents the $l$-th RQA measure. Therefore, $S^{(l)}$ can be split in $q$ samples
Table 3. RQA parameters.

\begin{tabular}{lc}
\hline Embedding & 10 \\
Radius & 80 \\
Line & 5 \\
Shift & 1 \\
Epoch & 50
\end{tabular}

Distance Meandist, Euclidean

No. of epochs 642

according to the rolling "window" $\mathcal{I}_{k, i}$ such that $S_{k, i}^{l}$ $=\left\{S_{t}^{l} \mid t \in \mathcal{I}_{k, i}\right\}$.

For each $l \neq m$, we denote $\rho_{l, m}$ as the Spearman's correlation coefficient between $S^{l}$ and $S^{m}$ and $\rho_{l, m}^{k, i}$ as the Spearman's correlation coefficient between $S_{k, i}^{l}$ and $S_{k, i}^{m}$. Therefore, there are $p=\left(\begin{array}{l}L \\ 2\end{array}\right)$ pair of correlations $\rho_{l, m}$ and $q \times p$ pair of "windows" correlations $\rho_{l, m}^{k, i}$ so that the product

$P_{a b s}(R Q E)_{k, i}=\prod_{\substack{l, m=1 \\ l \neq m}}^{L}\left(1+\left|\rho_{l, m}^{k, i}\right|\right)$

can be defined as the RQE (absolute) correlation index for the rolling window $\mathcal{I}_{k, i}$ and it varies between 0 and $2^{p}$.

\subsection{RQE correlation index on a test signal}

In order to check whether the above-mentioned correlation index can help in understanding the changes in a
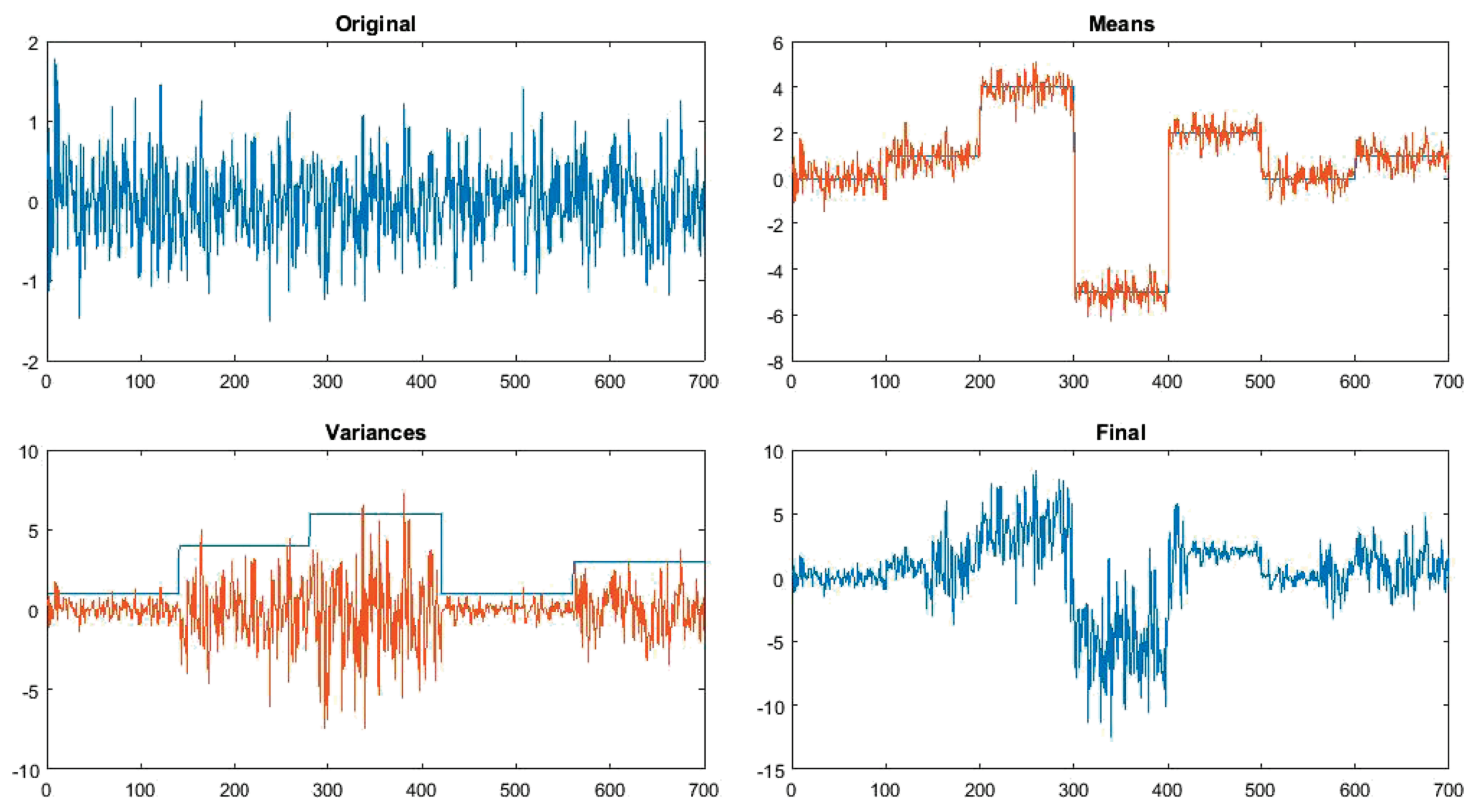

Figure 4. Clockwise: original signal, mean jumps, changes in variance and resulting final signal. 

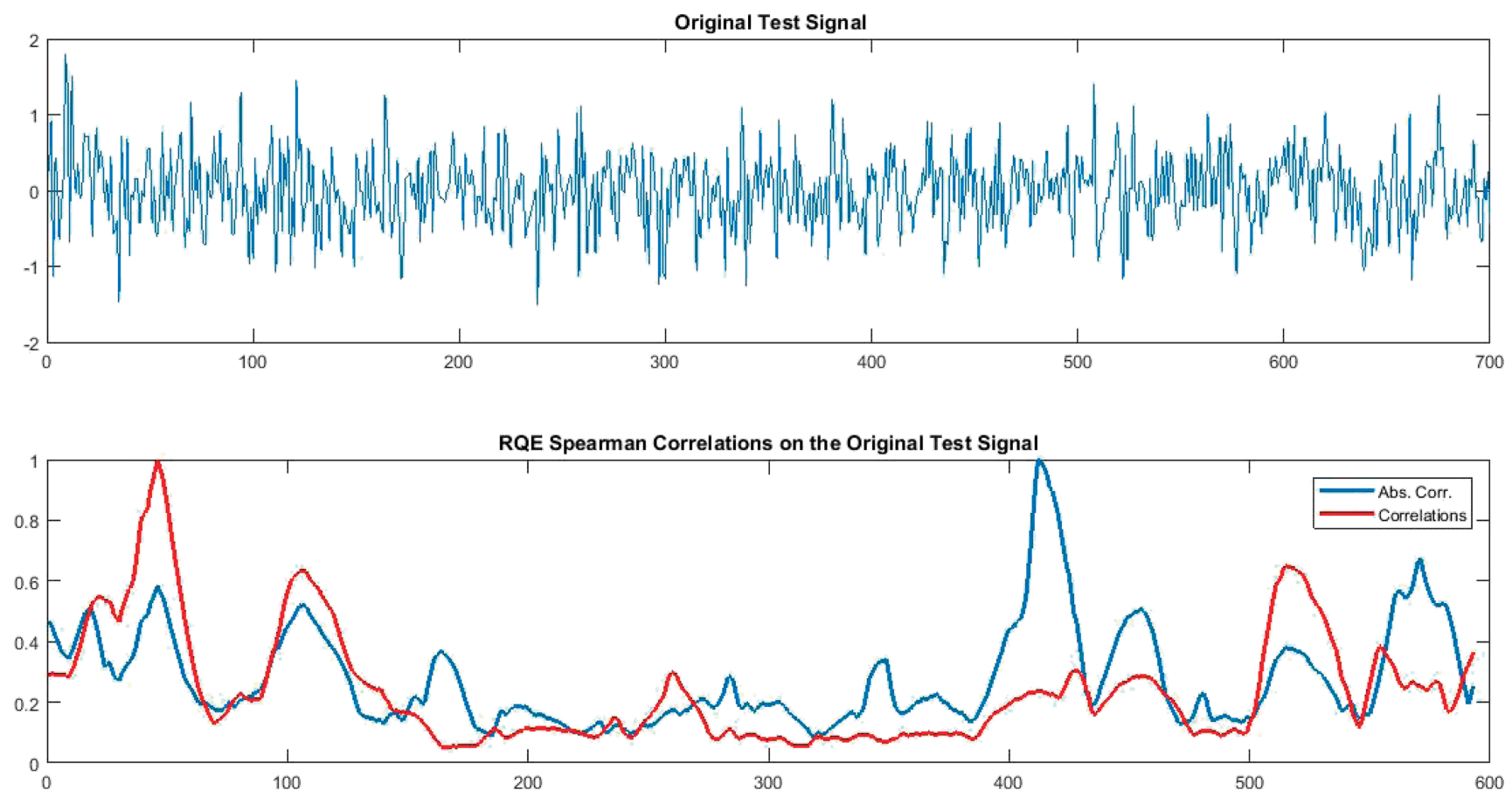

Figure 5. Spearman correlations (below) versus the original test signal (above). RQE absolute correlation (in blue) is displayed next to correlation (red). Difference in the x-axis numbering between the picture above and below, is due to the windowing mechanism.

times series we start with a known signal. Let us simulate a random signal normally distributed $\varepsilon \sim \mathcal{N}\left(\mu, \sigma^{2}\right)$ and let us perturb its mean and variance as in table 2 .
Now let us apply the RQA on both the original and the transformed signal by using the parameters in table 3 and shown in figure 4.
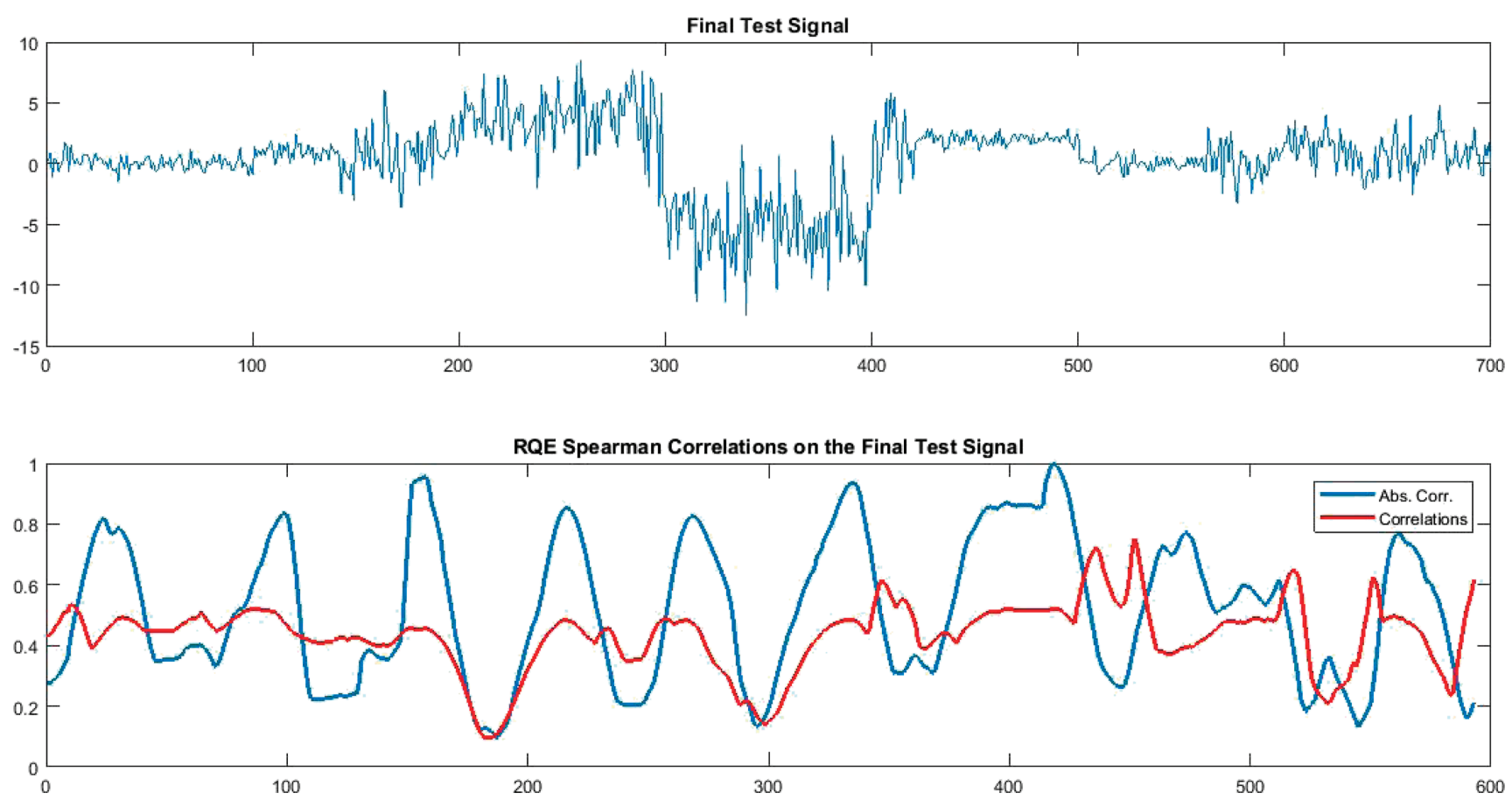

Figure 6. Spearman correlations (below) versus the final test signal (above). RQE absolute correlation (in blue) is displayed next to correlation (red). See how the RQE correlation calculated as in eq. (1) is closer than the other and it is able to detect more finely changes in the times series. Difference in the $\mathrm{x}$-axis between the picture above and below, is due to the windowing mechanism. 


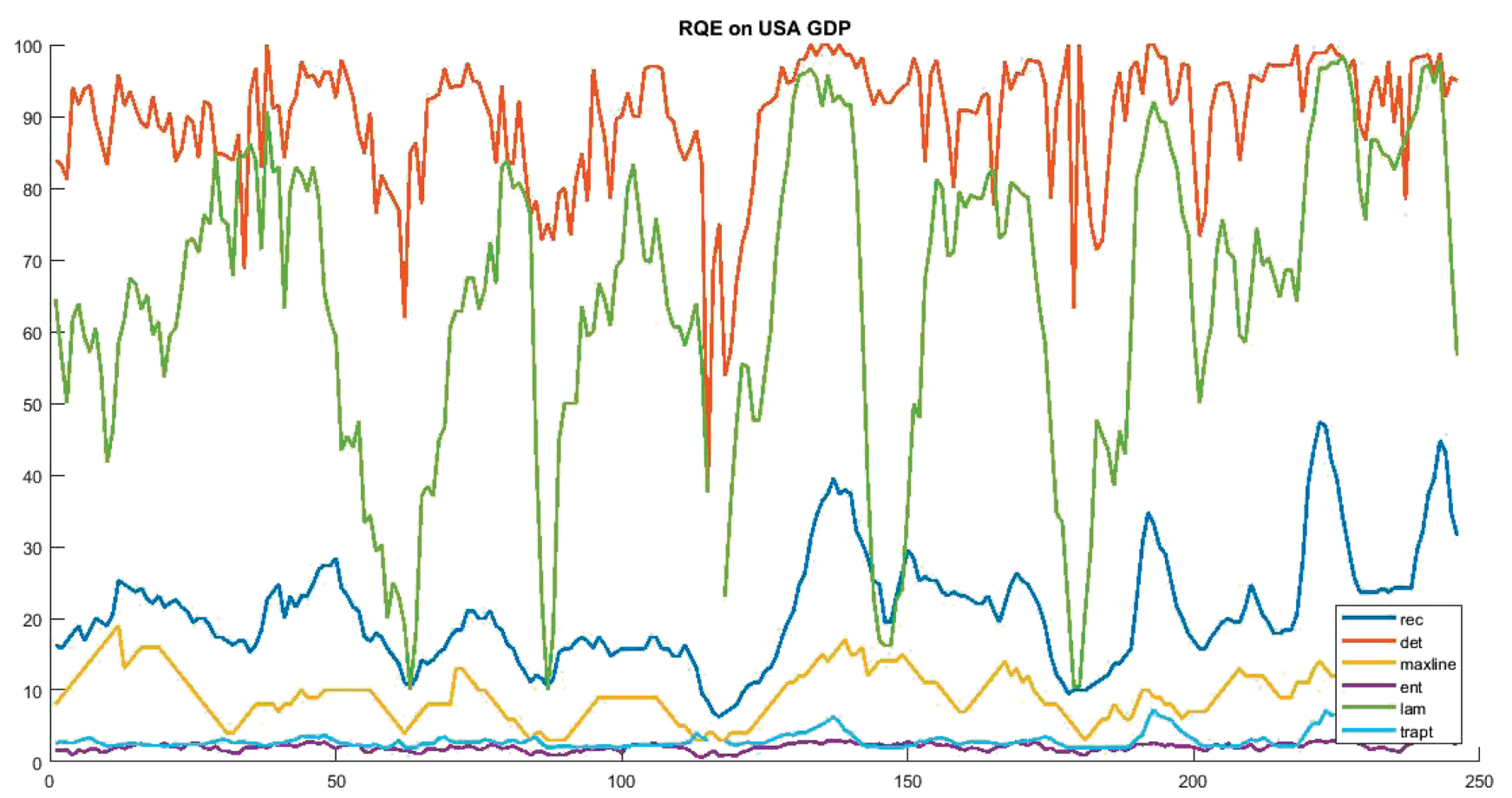

Figure 7. RQE indicators on USA (quarterly) GDP as retrieved from OECD database ("USA QGDP TOT PC_CHGPP Q").

The resulting correlation for the original signal and the final signal is displayed in figures 5 and 6 respectively. It is worth noting the fact that the RQE absolute correlation 1 is able to detect 9 of the 10 intervals in table 2 (one being clouded by the windowing filtering).
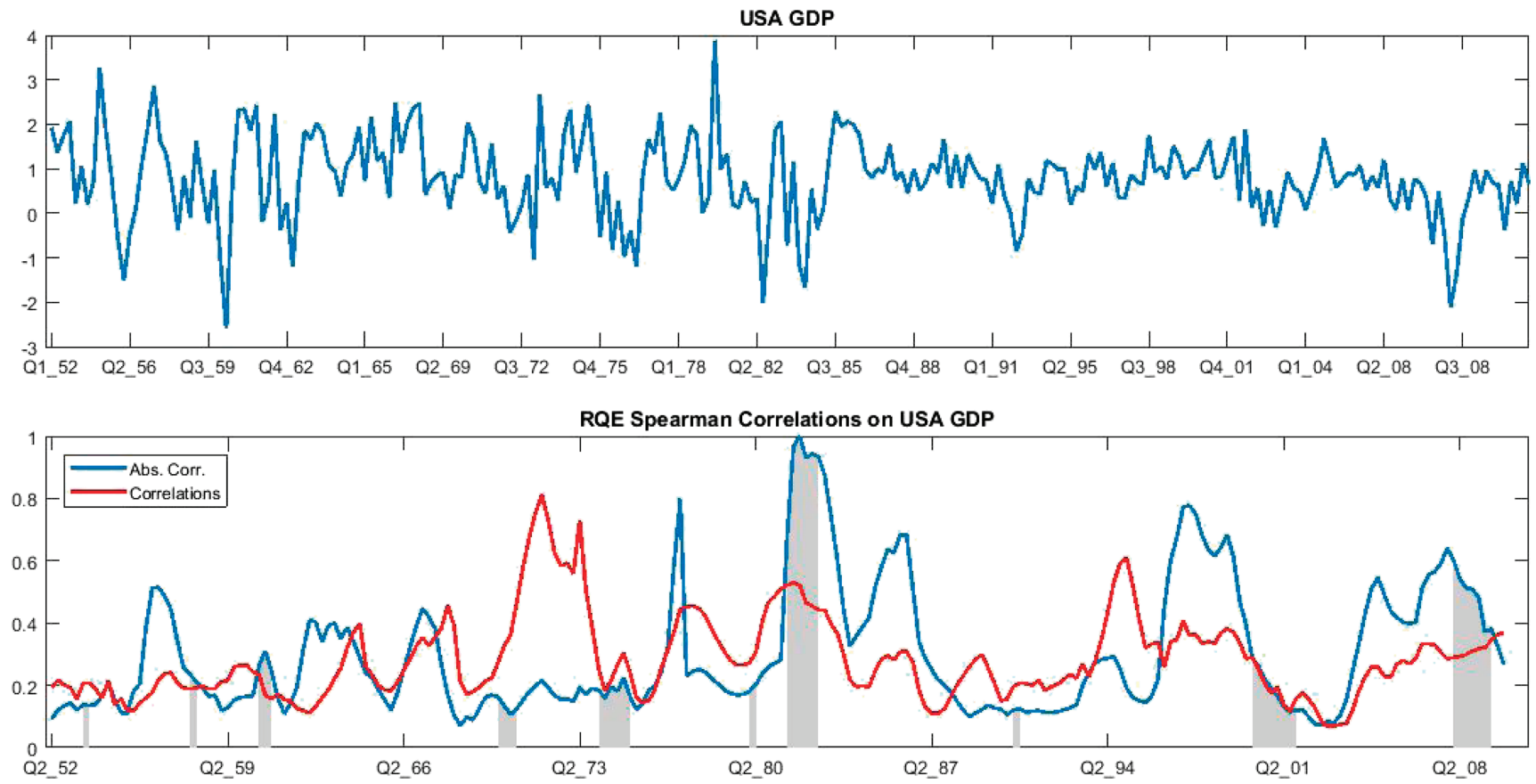

Figure 8. Maximum correlations (in blue) between RQE measures versus recession periods (in grey). As shown in the figure a change in the index is often linked to a recession. Spearman correlations (below) versus the final test signal (above). RQE absolute correlation (in blue) is displayed next to correlation (red). See how the RQE correlation calculated as in eq. (1) is more reactive than the other and it is able to detect more finely changes in the original times series. Difference in the $\mathrm{x}$-axis numbering between the picture above and below, is due to the windowing mechanism. 


\subsection{RQE correlation index on GDP data}

As we have shown in the previous section the RQE correlation index in eq. (1) can detect regimes' changes that are difficult to see at a glance. Therefore, an additional potential use of the index is as an early indicator in economics for recessions and market crashes, in seismology for earthquakes, etc.

To show an application to economics, we have retrieved from the OECD database the USA GDP [19] and then we have run a RQE on the data. In the following pictures we show first the set of RQE indicators on USA (quarterly) GDP changes as retrieved from OECD database figure 7 and second the Spearman correlation indices next to the USA GDP changes figure 8.

Finally, by considering the correlation among RQE measures (see figure 3), business cycles of 5-7 years were found (which is consistent with existing literature, e.g. Prescott (1986) [20] defines business cycles as 12-32 quarter cycles).

\section{Conclusion}

This work tested correlations of RQA on a simple signal and then investigated the application to business time series. RQA is meant to be an efficient and relatively simple tool in nonlinear analysis. As the technique permits the identification of sudden phase changes it may be suitable to study business cycles for, possibly, early detection of recessions. Here, we have illustrated how the so called RQE (absolute) correlation index is able to detect hidden changes in the signal. However, further research is required in order to capture the specific characteristics of macroeconomic times series which are more complex to study than financial indices. The final aim is to develop the said technique in order to match more closely economic downturns and potentially use it for early detection of recessions.

In future, through a new extensive data collection, RQA and PCA will be performed in order to assess the suitability of this technique to studying business time series. Moreover, RQA, PCA and statistical analysis will be applied on a Kaldor-Kalecki [21] model to see whether it can generate series comparable to the real ones.

\section{Acknowledgements}

The authors are grateful to the referees and to their colleagues Carlo Lucheroni (School of Science and Technologies, University of Camerino) and Edward Bace
(Middlesex University London). Special thanks go to Nicola Basile (Department of Mathematics, University of Bari) for his comments and helpful discussions.

\section{References}

[1] National Bureau of Economic Research. The NBER's recession dating procedure business cycle dating committee. January 2008

[2] G Zimatore, A R Fetoni, G Paludetti, M Cavagnaro, M V Podda and D Troiani, Med. Sci. Monit. 17, MT41 (2011)

[3] G Zimatore, G Garilli, M Poscolieri, C Rafanelli, F T Gizzi and M Lazzari, Chaos 27, 043101 (2017)

[4] P M Crowley, Eur. Phys. J. Spec. Top. 164, 67 (2008)

[5] A N Gorban, E V Smirnova and T A Tyukina, Phys. A 389, 3193 (2010)

[6] J P Zbilut, In Economics: Complex Windows, pages 91 (Springer, 2005)

[7] P M Crowley and A Schultz, Bank of Finland Research Discussion Paper (2010)

[8] S Karagianni and C Kyrtsou, Stud. Nonlinear Dyn. Econometrics 15 (2011)

[9] W-S Chen, Phys. A 390, 1332 (2011)

[10] K Moloney and S Raghavendra, In Topics in Numerical Methods for Finance, pages 177 (Springer, 2012)

[11] A Fabretti and M Ausloos, Int. J. Mod. Phys. B 16, 671 (2005)

[12] P M Addo, M Billio and D Guegan, N. Am. J. Sports Phys. Ther. 26, 416 (2013)

[13] F Strozzi, E Gutierrez, C Noè, T Rossi, M Serati and $\mathrm{J}$ Zaldivar, Application of non-linear time series analysis techniques to the nordic spot electricity market data (Libero istituto universitario Carlo, Cattaneo, 2007)

[14] O Piskun and S Piskun, arXiv preprint arXiv:1107.5420, (2011)

[15] J A Bastos and J Caiado, Physica A-statistical Mechanics and Its Applications - PHYSICA A 390, 04 (2011)

[16] E García-Ochoa, J González-Sánchez, N Acuña and J Euan, J. Appl. Electrochem. 39, 637 (2009)

[17] G Kousik, B Basabi and A. R Chowdhury, Phys. A 389, 1874 (2010)

[18] BEA. USA Recessions, Gross Domestic Product [A191RP1Q027SBEA] - US. Bureau of Economic Analysis, November 2016. Retrieved from FRED, Federal Reserve Bank of St. Louis; November 10, 2016

[19] OECD. Quarterly GDP (indicator). DOI: 10.1787/ b86d1fc8-en, 2016

[20] E C Prescott. Theory ahead of business-cycle measurement. In Carnegie-Rochester conference series on public policy, volume 25, pages 11 (Elsevier, 1986)

[21] G Orlando, Math. Comput. Simul. 125, 83 (2016) 